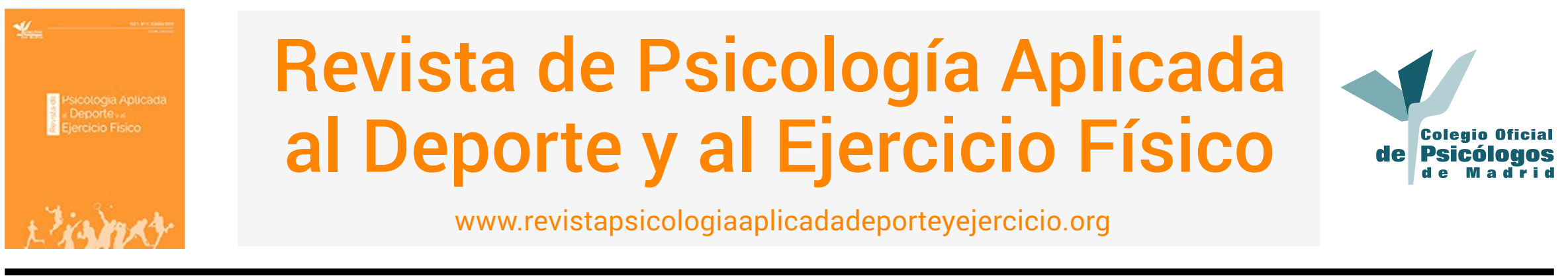

\title{
Entrenamiento psicológico y mejora de aspectos psicológicos relevantes para el rendimiento deportivo en jugadoras de fútbol
}

\author{
Aurelio Olmedilla', María Fuensanta Sánchez-Aldeguer², Clara M. Almansa², Verónica Gómez-Espejo³ y Enrique \\ Ortega $^{1}$

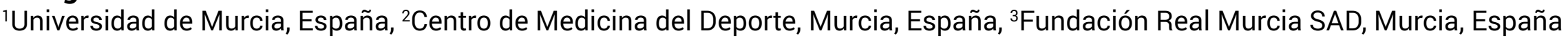

RESUMEN: El objetivo de este estudio fue determinar la eficacia de un programa de entrenamiento psicológico en equipos de fútbol femenino respecto a las características psicológicas relevantes para el rendimiento deportivo. Las participantes fueron 48 jugadoras de futbol de 3 equipos de Segunda División Nacional, con una media de edad de 21.15 \pm 3.89 y una media de años de experiencia de 12.48 \pm 4.12. Se utilizó el Cuestionario de Características Psicológicas relacionadas con el Rendimiento Deportivo (CPRD), tomando medidas pre-post intervención. Se agrupó a las participantes en Grupo Control (GC), Grupo Experimental 1 (GE1) y Grupo Experimental 2 (GE2). El programa de entrenamiento psicológico fue de 10 sesiones. Los resultados indican que no hay diferencias estadísticamente significativas entre los grupos GC y GE1 en ninguna de las escalas del CPRD. El GE2 presenta diferencias estadísticamente significativas en las escalas Influencia de la Evaluación del Rendimiento y tendencia a la significación en Control de Estrés (CE) del CPRD. Se concluye que las futbolistas que recibieron el programa de intervención psicológica (GE2) mejoraron significativamente sus recursos para gestionar mejor la evaluación que se hace de su rendimiento.

PALABRAS CLAVES: Entrenamiento psicológico, fútbol, mujeres, psicología, rendimiento.

Psychological training and improvement in psychological aspects relevant to sports performance in female footballers

ABSTRACT: The purpose of this study was to assess the effectiveness of a psychological training program regarding the psychological characteristics relevant to sports performance intended for women's football teams. The participants were 48 female football players from three teams of the Spanish second national division with a mean age of $21.15 \pm 3.89$ and a mean experience of $12.48 \pm 4.12$ years. The Questionnaire of Sports Performance-related Psychological Characteristics (QSPPC) was used, making measurements before and after intervention. The participants were assigned to a Control Group (CG), an Experimental Group 1 (EG1), and an Experimental Group 2 (EG2). The psychological training program consisted of 10 sessions. The results suggest that there are no statistically significant differences between the CG and GE1 groups in any of the QSPPC scales. The EG2 shows statistically significant differences in the Impact of Performance Evaluation scale and a trend to significance in the Stress Control (SC) scale of the questionnaire. It is concluded that the players who received the psychological intervention program (EG2) significantly improved their resources to better manage the evaluation of their performance.

KEYWORDS: Psychological training, football, women, psychology, performance.

Financiación: El trabajo ha sido realizado gracias al Proyecto Fútbol del Convenio entre la Federación de Fútbol de la Región de Murcia y la Universidad de Murcia (FFRM-UMU04 0092321 B 64502 14704)

Aurelio Olmedilla es Psicólogo en el Dpto. Personalidad, Evaluación y Tratamiento Psicológico. Universidad de Murcia. Campus Mar Nostrum; María Fuensanta Sánchez-Aldeguer es Psicóloga en el Centro de Medicina del Deporte. Universidad de Murcia. Campus Mar Nostrum; Clara M. Almansa es fisioterapeuta en el Centro de Medicina del Deporte. Universidad de Murcia. Campus Mar Nostrum; Verónica Gómez-Espejo es Psicóloga en la Fundación Real Murcia SAD. Universidad de Murcia. Campus Mar Nostrum; Enrique Ortega es Licenciado en CAFD. Facultad de Ciencias del Deporte. Universidad de Murcia. Campus Mar Nostrum.

La correspondencia sobre este artículo debe enviarse al primer autor al e-mail: olmedilla@um.es 


\section{Formação psicológica e melhoria de aspetos psicológicos relevantes para o desempenho desportivo em jogadoras de futebol}

RESUMO: O objetivo deste estudo foi determinar a eficácia de um programa de treino psicológico em equipas de futebol feminino relativamente às características psicológicas relevantes para o desempenho desportivo. As participantes foram 48 jogadoras de futebol de 3 equipas da Segunda Divisão Nacional, com uma idade média de 21,15 \pm 3,89 e uma média de anos de experiência de 12,48 \pm 4,12. 0 questionário de características psicológicas relacionadas com o desempenho desportivo (CPRD) foi utilizado, tomando medidas pré e pós-intervenção. Agruparam-se as participantes entre o Grupo de Controlo (GC), o Grupo Experimental 1 (GE1) e o Grupo Experimental 2 (GE2). O programa de treino psicológico foi de 10 sessões. Os resultados indicam que não há diferenças estatisticamente significativas entre os grupos GC e GE1 em qualquer uma das escalas do CPRD. O GE2 apresenta diferenças estatisticamente significativas nas escalas Influência da Avaliação do Desempenho e tendência à significação em Controlo de Stress (CS) do CPRD. Conclui-se que as jogadoras que receberam o programa de intervenção psicológica (GE2) melhoraram significativamente os seus recursos para gerir melhor a avaliação feira do seu desempenho.

PALAVRAS-CHAVE: Treino psicológico, futebol, mulheres, psicologia, desempenho.

Artículo recibido: 20/01/2018 | Artículo aceptado: 08/03/2018

El factor psicológico es un tópico que ha ido tomando fuerza en la descripción de las facultades que debe tener un deportista. Es más, este factor es considerado como mediador entre las capacidades físicas, técnicas y tácticas de los deportistas por lo que la disposición psicológica de éstos determinará su funcionamiento deportivo, afectando a su rendimiento en las competiciones (Mahamud, Tuero y Márquez, 2005; Pazo y Sáenz-López, 2008). Así, el papel del factor psicológico en el rendimiento deportivo ha sido constatado en múltiples investigaciones (Abdullah, Musa, Maliki, Kosni y Suppiah, 2016; Castilla y Ramos, 2012; Morris, 2000). En consecuencia, debe ser considerado e integrado en el entrenamiento, para alcanzar el mejor rendimiento deportivo posible (González, 2017; Olmedilla y Domínguez-Igual, 2016).

Algunos autores indican que muchos deportistas y entrenadores consideran que la eficacia en el deporte viene determinada por el aspecto mental, entre el $40 \%$ y el $90 \%$ de las veces, dependiendo del deporte y de la situación o momento determinado (Martínez-Ferreiro, 2016). Estudios clásicos encontraron que las variables fisiológicas explicaban entre el $45 \%$ y el $48 \%$ del rendimiento deportivo, pero cuando se unían las variables psicológicas llegaban a explicar entre el $79 \%$ y el $85 \%$ del éxito en la especialidad de lucha (Nagle, Morgan, Hellickson, Serfass y Alexander, 1975; Silva, Shultz, Haslam y Murray, 1981).

En esta línea, hay investigaciones que aportan información sobre qué variables psicológicas se deben considerar para valorar y mejorar el desarrollo óptimo. Williams y Reilly (2000) destacan la autoconfianza, el control de la ansiedad, la motivación y la concentración. Junichi y Hajime (2007) hacen énfasis en aspectos como el control del estrés o la reducción de la ansiedad, señalando incluso que en todas las áreas de funcionamiento que implica el deporte de com- petición intervienen dichas variables psicológicas. Otros estudios se han centrado en el análisis de la relación entre rasgos de personalidad y rendimiento deportivo (Cabrita, Rosado, de la Vega y Serpa, 2014; Orlick, 2003; Pazo, Sáenz-López y Fradua, 2012; Torres-Luque, Hernández- García, Olmedilla, Ortega y Garatachea, 2013), destacando el estudio de Gee, Dougan, Marshall y Dunn (2007) donde rasgos como la competitividad, la orientación de equipo, la autoconfianza y la disposición analítica aparecen como predictores significativos del rendimiento deportivo. Tal y como señalan González-Campos, Valdivia-Moral, Zagalaz y Romero (2015) los aspectos psicológicos conforman una estructura importante para el desarrollo de las capacidades del deportista, que pueden contribuir a optimizar el rendimiento deportivo (Fernández-Fernández, Sánchez-Sánchez y Zurita, 2013; Mora, Zarco y Blanca, 2001).

En este sentido, la evaluación de las habilidades psicológicas de los deportistas permitir establecer hipótesis de trabajo acerca de la intervención psicológica más apropiada para favorecer el rendimiento deportivo (Abenza et al., 2014; Gimeno, Buceta y Pérez-Llantada, 2007; Olmedilla, García-Mas y Ortega, 2017; Olmedilla, Ortega, Andreu y Ortín, 2010). Conocer el perfil psicológico de un deportista permite comprenderlo mejor, mejorar los procesos de comunicación con el mismo y aumentar la efectividad de los entrenamientos (Martínez-Ferreiro, 2016), lo que redundará en una mejor preparación mental y en adquisición de habilidades psicológicas que distinguen al deportista de éxito del resto (Bahrololoum, Hassani, Reza y Akbari, 2012).

Así pues, el entrenamiento psicológico se puede considerar como una forma más del entrenamiento deportivo (García-Naveira y Jerez, 2012; Jaenes, Caracuel y Penaloza, 2012; Lorenzo, Pujals, Navarro y Lorenzo, 2012), que incide directa- 
mente en los procesos de formación de los deportistas y ha de basarse, por un lado, en el aprendizaje de habilidades y estrategias psicológicas que permitan el afrontamiento más adecuado de las diferentes situaciones deportivas (Cantón, Checa y Ortin, 2009; Reyes, Raimundi y Gómez, 2012), y por otro, en el fomento del bienestar psicológico del deportista que le permita crecer y madurar como persona (Olmedilla, Bazaco, Ortega y Boladeras, 2011; Romero et al., 2010). La mayoría de programas de entrenamiento psicológico en el deporte se basan en el marco teórico de la Terapia Cognitivo Conductual (Brawley, Rejeski y Lutes, 2000; Lindern, 2016; Mahoney, 2013; Mostaan, Sanatkaran y Bahari, 2015), considerando además los principios clave de la misma en este campo de trabajo (McArdle y Moore, 2012), y aplicados en diferentes contextos deportivos (Guerrero, Saldaña, Bulnes, del Castillo y Palencia, 2017; Llames y García-Dantas, 2017; Olmedilla, Rubio, Ortega y García-Mas, 2017).

Y aunque las propuestas de programas son básicamente iguales en jugadores que en jugadoras (Martín-García, 2003), es interesante tener en cuenta que las características psicológicas de unos y otras pueden diferir, tal y como indican Junge y Feddermann-Demont (2016) en su estudio sobre la prevalencia de depresión y ansiedad en jugadores de fútbol de alto nivel (mujeres y hombres), en el que los jugadores tenían una depresión y una ansiedad promedio significativamente menor que las jugadoras. Por su parte, Olmedilla, García-Alarcón y Ortega (en prensa) encuentran que las jugadoras lesionadas manifiestan niveles de estrés menores que las jugadoras no lesionadas, hallazgos que apuntan en una dirección contraria a los postulados del modelo de estrés y lesión de Andersen y Williams (1988), y de la bibliografía existente realizada mayoritariamente con muestras de deportistas masculinos.

En los últimos años han aumentado los trabajos que muestran interés en el estudio de la relación entre factores psicológicos y rendimiento deportivo en el fútbol femenino, destacando aspectos como la dureza mental, la autoestima y el control de la ansiedad (Williams, 2017), la predicción de las orientaciones de meta sugiriendo acciones específicas del entrenador (Granero-Gallegos, Gómez-López, Abraldes y Baena-Extremera, 2015), o el estudio de indicadores de salud mental como la depresión y la ansiedad (Junge y Feddermann-Demont, 2016), o los estados de ánimo (Arroyo del Bosque, Irazusta y González-Rodríguez, 2016). Así, parece muy pertinente la sugerencia de Rutkowska y Bergier (2015) señalando que examinar el perfil psicológico de las jugadoras puede otorgar mucha información útil para mejorar los programas de entrenamiento, especialmente en entrenamiento mental y en jugadoras que se aproximan al nivel profesional, tal y como plantea Martín-García (2003).
El objetivo de este estudio fue determinar la eficacia de un programa de entrenamiento psicológico en equipos de fútbol femenino respecto a las características psicológicas relevantes para el rendimiento deportivo. La hipótesis a plantear es la siguiente: "Las jugadoras que reciban entrenamiento psicológico mejorarán sus recursos y habilidades psicológicas frente a aquellas que no realicen este entrenamiento".

\section{Método}

\section{Participantes}

Los participantes fueron 48 jugadoras de fútbol, de las cuales 11 pertenecientes a 3 equipos de la categoría deportiva de Segunda División Nacional. Por razones de proximidad geográfica y viabilidad del estudio, 2 equipos (equipo 1 y equipo 2) fueron asignados al Grupo Experimental (GE1 y GE2) y el equipo 3 lo fue al Grupo Control (GC).

En la Tabla 1 se presenta una descripción de la muestra en función a la edad y años de experiencia.

Tabla 1. Edad y años de experiencia por equipos de la muestra

\begin{tabular}{cccc}
\hline Equipos & Jugadoras & Media Edad & Media Años experiencia \\
\hline 1-GE1 & 15 & $18.67 \pm 1.5$ & $10.53 \pm 3.18$ \\
2-GE2 & 14 & $21.64 \pm 4.68$ & $12.79 \pm 4.81$ \\
3-GC & 19 & $22.74 \pm 3.74$ & $13.79 \pm 3.82$ \\
Total & 48 & $21.15 \pm 3.89$ & $12.48 \pm 4.12$ \\
\hline
\end{tabular}

\section{Instrumentos}

Se utilizó el Cuestionario de Características Psicológicas del Rendimiento Deportivo (CPRD) de Gimeno et al. (2001) para las evaluación de las variables psicológicas relevantes. El CPRD está compuesto por 55 Items en escala Likert de cinco puntos (de totalmente en desacuerdo a totalmente de acuerdo) con una opción de respuesta adicional para aquellos casos en los que el deportista «no entiende el ítem», con el fin de evitar respuestas «en blanco» o en la posición central. Presenta confiabilidad y validez con una consistencia interna del cuestionario en su conjunto de .85 .

El CPRD contiene 5 factores: Control de Estrés (CE) ( $a=.88)$, Influencia de la Evaluación del Rendimiento (IER) ( $a=.72)$, Motivación (MO) $(\mathrm{a}=.67)$, Habilidad Mental (HM) $(\mathrm{a}=$.34) y Cohesión de Equipo $(\mathrm{CH})(\mathrm{a}=.78)$. La baja consistencia interna del factor HM, según Gimeno et al. (2001), es posible que se deba 
al hecho de que el conjunto de ítems que la integran no represente un único y completo constructo. Sin embargo, explican que la agrupación de sus ítems en un factor con saturaciones superiores a .30, justifica que se mantenga en el cuestionario CPRD y se considere la información que aporta.

La escala CE está formada por 20 ítems y hace referencia a situaciones potencialmente estresantes en las que es necesario el control, y a las respuestas del deportista en relación a las demandas del entrenamiento y la competición. Una puntuación alta indica que el deportista dispone de recursos psicológicos para controlar el estrés relacionado con su práctica deportiva. La escala IER está formada por 12 ítems, y hace referencia a las características de las respuestas del deportista ante situaciones en las que éste evalúa su propio rendimiento, o se plantea que lo están evaluando personas significativas a él; además, incluye también la valoración respecto a los antecedentes que pueden originar una valoración del rendimiento deportivo del deportista. Una puntuación alta indica que el deportista muestra un elevado control del impacto de una evaluación negativa sobre su rendimiento. La escala MO está formada por 8 ítems y hace referencia a la motivación básica por el rendimiento y logros deportivos, y a la motivación cotidiana por el entrenamiento diario o la competición. Una puntuación alta indica que el deportista se encuentra muy motivado para la práctica deportiva competitiva. La escala HM está formada por 9 ítems y hace referencia al uso de una serie de habilidades que pueden repercutir en el funcionamiento del deportista en beneficio de su rendimiento. Una puntuación alta indica que el deportista dispone de unos recursos que pueden ayudarle a rendir mejor. La escala $\mathrm{CH}$ está formada por 6 reactivos y hace referencia al grado en que el deportista se siente atraído e identificado con el grupo deportivo. Una puntuación alta indica que el deportista está integrado y presenta una buena disposición a trabajar con el equipo.

\section{Procedimiento}

Se implementó un programa de intervención psicológica, cuyo marco teórico es el más utilizado en la aplicación profesional de la psicología del deporte, basado en la Terapia Cognitivo Conductual, considerando los principios clave de la misma en este campo de trabajo (McArdle y Moore, 2012) y con uno de los niveles de eficacia más importantes (Hofmann, Asnaani, Vonk, Sawyer y Fang, 2012; Olatunji, Cisler y Deacon, 2010)

La encargada de realizar el programa fue una graduada en psicología, máster en Psicología General Sanitaria y formación específica en psicología de la actividad física y del deporte, con dos años de experiencia.

El programa de intervención psicológica fue el mismo para los dos grupos experimentales, adecuándose en cada caso a las fechas de disponibilidad para las sesiones, si bien en ambos casos el cronograma propuesto se cumplió. Se realizaron 10 sesiones grupales (solo las jugadoras y la psicóloga) de 1 hora de duración aproximadamente, cada 15 días, antes de comenzar el entrenamiento deportivo y en los vestuarios de cada uno de los equipos. En la Tabla 2 se puede observar el cronograma y contenidos del programa.

Tabla 2. Cronograma y contenidos generales del programa

\begin{tabular}{lll}
\hline Diciembre & Sesión 1 & Evaluación Inicial \\
Enero & Sesión 2 & Psicoeducación \\
Enero & Sesión 3 & Establecimiento de \\
Febrero & Sesión 4 & objetivos \\
Febrero & Sesión 5 & Reestructuración \\
Marzo & Sesión 6 & cognitiva \\
Marzo & Sesión 7 & Reestructuración \\
Abril & Sesión 8 & cognitiva \\
Mayo & Sesión 9 & Relajación \\
Mayo & Sesión 10 & Relajación \\
& & Visualización \\
& & Visualización \\
& & Evaluación Final \\
\hline
\end{tabular}

Tabla 3. Contenidos específicos del programa

1. Formación psicológica: Psicoeducación

1.1. Motivación: es lo que nos mueve a realizar cualquier conducta, tanto a iniciarla como a mantenerla. Se trata de fomentar la motivación intrínseca, la cual procede del propio individuo.

1.1.1. Técnicas: Es importante el establecimiento de objetivos sencillos y realistas para tener una meta que lograr y sentirse realizado con ella Para ello estableceremos objetivos a corto (1 día, 1 semana, 1 mes) y largo plazo (1 temporada, 1 año).

Carteles motivacionales: colocamos en el vestuario carteles con frases motivadoras que lean en cada entrenamiento y en cada partido, de manera que las tengan presentes 
1.2. Autoconfianza: creer que puedo hacer lo que me proponga, seguridad en mí mismo y autoconocimiento personal.

1.3. Autoeficacia: expectativa de que obtendré un resultado exitoso debido a mi esfuerzo y los recursos que tengo para ello.

1.4. Cohesión Grupal: en los deportes de equipo es fundamental la participación de cada miembro del mismo.

1.4.1. Aspectos relevantes: Espíritu de equipo: mejora la cooperación, mejor entendimiento y con ello mejor rendimiento en estrategias de juego, favorece un clima positivo y beneficioso (que ayuda a mantener una baja ansiedad), y sobre todo lleva a la consecución de objetivos de manera más eficaz.

1.5. Evaluación de las personas relevantes: debemos tener en cuenta las opiniones de las personas importantes para nosotros pero no debe prevalecer sobre lo que pensemos nosotros mismos, sobre todo cuando hablamos de nuestro esfuerzo y nuestro rendimiento.

2. Establecimiento de objetivos

2.1. Explicación

2.1.1. Objetivos a corto, medio y largo plazo

2.1.2. Objetivos de rendimiento y de resultado

2.1.3. Objetivos individuales y colectivos

2.2. Ejercicios

2.2.1. Tabla registro de objetivos.

3. Reestructuración cognitiva

\subsection{Explicación}

3.1.1. El porqué de la técnica y su funcionamiento

3.2. Ejercicios reestructuración cognitiva

3.2.1. Sustitución de pensamientos negativos como "no puedo" por "poco a poco lo conseguiré", "soy capaz", por otros más positivos.

3.2.2. Detención del pensamiento: si pensamos algo negativo durante el partido o el entrenamiento diremos "ino!" o "ibasta!"

3.2.3. Focalización: no distraernos con otras cosas durante el entrenamiento o partido.

4. Aprendizaje de técnicas: Relajación

4.1. Explicación

4.1.1. El porqué de la técnica y su funcionamiento

4.2. Ejercicios relajación-respiración

4.2.1. Respiración en 4 tiempos: boca-garganta-pecho-estómago.

4.2.2. Relajación de 4 grupos musculares:

$1{ }^{\circ}$ Grupo: brazos.

$2^{\circ}$ Grupo: cara-cuello

$3^{\circ}$ Grupo: espalda-hombros-abdomen.

$4^{\circ}$ Grupo: piernas.

4.2.3. Justo antes del partido: durante un minuto cerrar los ojos, sentadas en los banquillos con las piernas sobre las rodillas y mientras respiran profundamente (4 tiempos) pensar en la victoria en el partido.

5. Aprendizaje de técnicas: Visualización

5.1. Explicación

5.1.1. El porqué de la técnica y su funcionamiento

5.2. Ejercicios visualización

5.2.1. Cuestionario Visualización Deportiva.

5.2.2. Visualización deportiva general y específica: entrenar/jugar bien, concentrarnos en las buenas sensaciones. 
Todas las jugadoras fueron contactadas a través de sus clubes de procedencia organizando una reunión en la que se les explicó el objetivo del estudio y firmaron un formulario de consentimiento informado.

\section{Diseño}

Diseño cuasi-experimental de intervención pre-post. Muestreo incidental.

\section{Análisis Estadístico}

Se realizó un análisis estadístico descriptivo, mediante el uso de medias y desviaciones típicas. Con el objeto de poder analizar las diferencias entre las medidas iniciales y finales en cada una de las variables y tras al comprobación de la normalidad de los datos, se utilizó la prueba T de Student para dos muestras relacionadas. Se utilizó un nivel de significación de $p<.05$. Los datos fueron analizados con el programa estadístico Spss. V. 21.

\section{Resultados}

En la Tabla 4 se pueden observar las puntuaciones medias de las jugadoras en cada uno de los factores del CPRD, tanto antes del programa de entrenamiento psicológico como después del mismo.

Tabla 4. Puntuaciones pre-post en los factores del CPRD

\begin{tabular}{|c|c|c|c|c|c|c|}
\hline & \multicolumn{2}{|c|}{ GE1 } & \multicolumn{2}{|c|}{ GE2 } & \multicolumn{2}{|c|}{ GC } \\
\hline & Media & D.T. & Media & D.T. & Media & D.T. \\
\hline Control Estrés Pre & 49.27 & 10.24 & 42.07 & 11.59 & 42.07 & 11.59 \\
\hline Control Estrés Post & 52.36 & 17.60 & 48.14 & 14.55 & 48.14 & 14.55 \\
\hline IER Pre & 26.33 & 6.85 & 23.57 & 6.06 & 23.57 & 6.06 \\
\hline IER Post & 28.91 & 9.84 & 28.71 & 9.71 & 28.71 & 9.71 \\
\hline Motivación Pre & 21.47 & 6,91 & 21.86 & 4.87 & 21.86 & 4.87 \\
\hline Motivación Post & 23.45 & 2.84 & 19.43 & 5.19 & 19.43 & 5.19 \\
\hline Habilidad Mental Pre & 19.93 & 5.42 & 22.21 & 3.96 & 22.21 & 3.96 \\
\hline Habilidad Mental Post & 21.27 & 2.57 & 20.86 & 3.24 & 20.86 & 3.24 \\
\hline Cohesión de Equipo Pre & 20.00 & 4.02 & 19.57 & 2.98 & 19.57 & 2.98 \\
\hline Cohesión de Equipo Pre & 21.18 & 1.83 & 18.00 & 3.37 & 18.00 & 3.37 \\
\hline
\end{tabular}

En la Figura 1 se pueden observar las puntuaciones centiles obtenidas por las jugadoras según los baremos del CPRD.

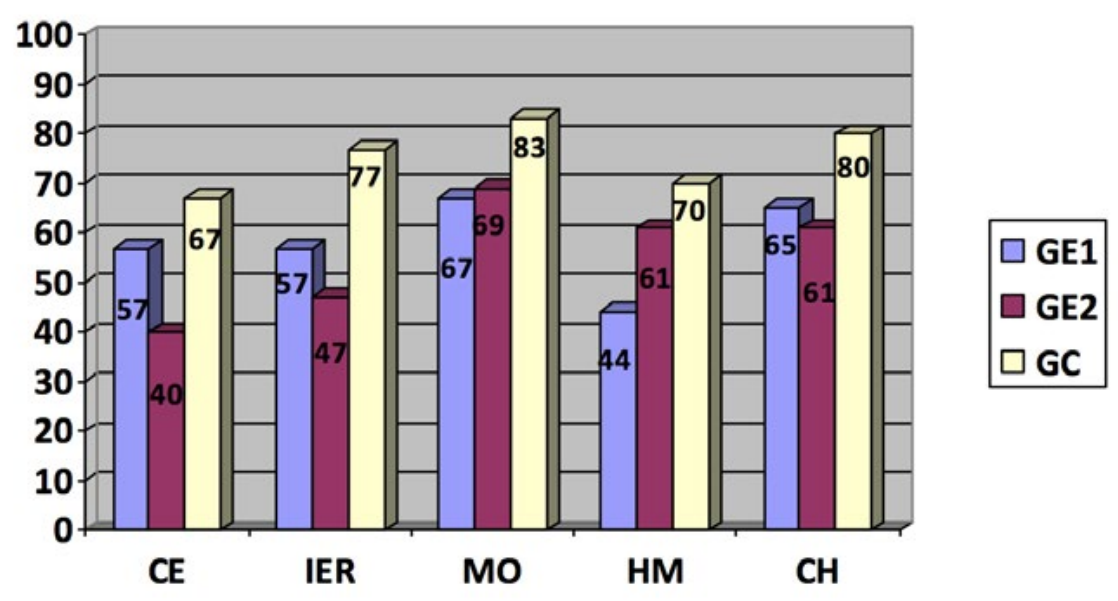

Figura 1. Puntuaciones centiles en el CPRD al inicio del programa de intervención psicológica.

Cabe destacar que las puntuaciones del GC están en las 5 escalas por encima del centil 60 , incluso alguna ( $\mathrm{MO}$ y $\mathrm{CH})$ en 80 o más. Sin embargo, las puntuaciones obtenidas por las jugadoras del GE1 como del GE2 se encuentran en un nivel medio, o medio bajo.

En la Figura 2 se pueden observar las puntuaciones centiles obtenidas por las jugadoras después del programa según los baremos del CPRD.

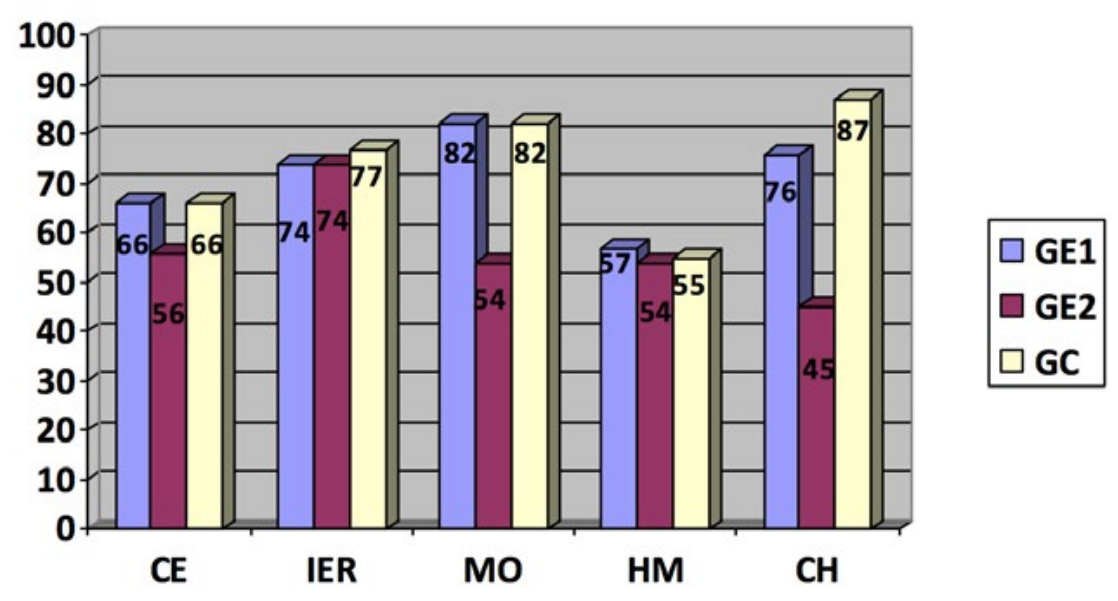

Figura 2. Puntuaciones centiles en el CPRD al finalizar el programa de intervención psicológica.

Cabe destacar que las puntuaciones del GC se mantienen igual o bajan (CE, IER, MO y HM), y solamente aumenta en $\mathrm{CH}$. Sin embargo, en el GE1 todas las puntuaciones centiles aumentan, y en el GE2 solo aumentan CE y IER.

En la Tabla 5 se muestran los datos de la prueba de muestras relacionadas del GE1 y su significación estadística en cada una de las parejas de los factores del CPRD.

Tal y como se puede apreciar no aparecen diferencias estadísticamente significativas en ninguno de los factores del CPRD. 
Tabla 5. Prueba de muestras emparejadas del equipo GE1

\begin{tabular}{lccccc}
\hline & \multicolumn{5}{c}{ Diferencias emparejadas } \\
\cline { 2 - 6 } & Media & D.T. & $t$ & gl. & Sig. (bilateral) \\
\hline $\begin{array}{l}\text { CE Pre } \\
\text { CE Post }\end{array}$ & -3.54 & 14.50 & -.811 & 10 & .436 \\
\hline $\begin{array}{l}\text { IER Pre } \\
\text { IER Post }\end{array}$ & -4.09 & 8.25 & -1.644 & 10 & .131 \\
\hline MO Pre & -2.54 & 6.84 & -1.233 & 10 & .246 \\
MO Post & -.36 & 4.73 & -.255 & 10 & .804 \\
\hline HM Pre & -1.81 & 4.33 & -1.392 & 10 & .194 \\
HM Post & & & & & \\
\hline CH Pre & & & & \\
CH Post & &
\end{tabular}

En la Tabla 6 se muestran los datos de la prueba de muestras emparejadas del GE2 y su significación estadística en cada una de las parejas de los factores del CPRD.

Tabla 6. Prueba de muestras emparejadas del equipo GE2

\begin{tabular}{|c|c|c|c|c|c|}
\hline & \multicolumn{5}{|c|}{ Diferencias emparejadas } \\
\hline & Media & D.T. & $t$ & gl. & Sig. (bilateral) \\
\hline $\begin{array}{l}\text { CE Pre } \\
\text { CE Post }\end{array}$ & -8.28 & 10.35 & -.2 .117 & 6 & .079 \\
\hline $\begin{array}{l}\text { IER Pre } \\
\text { IER Post }\end{array}$ & -4.00 & 3.65 & $-2,898$ & 6 & .027 \\
\hline $\begin{array}{l}\text { MO Pre } \\
\text { MO Post }\end{array}$ & 1.00 & 8.86 & $-2,898$ & 6 & .776 \\
\hline $\begin{array}{l}\text { HM Pre } \\
\text { HM Post }\end{array}$ & 2.71 & 5.93 & 1,210 & 6 & .272 \\
\hline $\begin{array}{l}\text { CH Pre } \\
\text { CH Post }\end{array}$ & 2.14 & 3.57 & 1,584 & 6 & .164 \\
\hline
\end{tabular}

Tal y como se puede apreciar en el factor IER aparecen diferencias estadísticamente significativas entre las puntuaciones pre y post $(p<.05)$. Además, se puede considerar la tendencia a la significación estadística en el factor CE ( $p$ $<.10)$.

En la Tabla 7 se muestran los datos de la prueba de muestras relacionadas del GC y su significación estadística en cada una de las parejas de los factores del CPRD.
Tabla 7. Prueba de muestras emparejadas del equipo GC

\begin{tabular}{lccccc}
\hline & \multicolumn{5}{c}{ Diferencias emparejadas } \\
\cline { 2 - 6 } & Media & D.T. & $t$ & gl. & Sig. (bilateral) \\
\hline $\begin{array}{l}\text { CE Pre } \\
\text { CE Post }\end{array}$ & -1.00 & 2.94 & -.679 & 3 & .546 \\
\hline $\begin{array}{l}\text { IER Pre } \\
\text { IER Post }\end{array}$ & -3.25 & 4.19 & 1.550 & 3 & .219 \\
\hline MO Pre & 1.00 & 1.82 & 1.095 & 3 & .353 \\
MO Post & 2.00 & 4.69 & .853 & 3 & .456 \\
\hline HM Pre & & & 3 & \\
HM Post & .25 & .95 & .522 & 3 & .638 \\
\hline $\begin{array}{l}\text { CH Pre } \\
\text { CH Post }\end{array}$ & & & & &
\end{tabular}

Tal y como se puede apreciar no aparecen diferencias estadísticamente significativas en ninguno de los factores del CPRD.

\section{Discusión}

El objetivo de este estudio fue determinar la eficacia de un programa de entrenamiento psicológico en equipos de fútbol femenino respecto a las características psicológicas relevantes para el rendimiento deportivo. Los resultados muestran que el programa aplicado es efectivo respecto a las dos escalas relacionadas con la gestión del estrés (CE y IER) en ambos grupos experimentales, aunque solo en el GE2 las diferencias son estadísticamente significativas; estos resultados están en la línea de lo indicado por estudios clásicos (Jones, 1993; Whelan, Mahoney y Meyers, 1991) respecto a la efectividad de programas cognitivo-conductuales.

Respecto a la hipótesis planteada, "las jugadoras que reciban entrenamiento psicológico mejorarán sus recursos y habilidades psicológicas frente a aquellas que no realicen este entrenamiento", los resultados parecen confirmar ésta. EI GE1 aumenta sus puntuaciones en todas las escalas del CPRD, y el GE2 lo hace en dos de las escalas, y en este caso además con diferencias estadísticamente significativas; incremento que no se produce en el GC. Estos resultados están en la línea de lo encontrado por otros autores (Bennett y Maynard, 2016; Olmedilla y Domínguez-Igual, 2016; OlmediIla, Ortega, González y Serpa, 2015), resaltando la eficacia de sus intervenciones psicológicas, incluso desde modelos teóricos diferentes al cognitivo-conductual (Gross et al., 2016).

Los resultados muestran que no hay diferencias estadísticamente significativas en los grupos GC y GE1 en ninguna 
de las escalas del CPRD, sin embargo, el grupo GE2 presenta diferencias estadísticamente significativas en IER y tendencia a la significación en CE. Por otro lado, al observar las puntuaciones centiles de las futbolistas de los tres equipos, éstas están, en general, en la línea de lo encontrado con futbolistas varones (Olmedilla, García-Mas y Ortega, 2017), si bien parece que éstos controlan mejor el estrés propio de la competición, es decir tienen mejores puntuaciones en la escala CE (82.5). En cualquier caso, las jugadoras del GE1 aumentan sus puntuaciones en las 5 escalas, por lo que se puede considerar que han mejorado su capacidad de controlar el estrés propio de la práctica deportiva, la mejor gestión del impacto de la evaluación de su rendimiento, la motivación, la cohesión de equipo y las habilidades mentales, características psicológicas todas ellas relevantes para el rendimiento deportivo. Por su parte el GE2 solo aumenta sus puntuaciones en CE y en IER, aunque de forma significativa, ya que aparece una tendencia estadística a la significación en la primera y significación estadística en la segunda. Quizá, el descenso en $\mathrm{MO}$ y $\mathrm{CH}$ se deba a la pérdida de objetivos deportivos al final de temporada, y el descenso en HM pueda deberse a cierto desencanto por lo anterior, lo que resalta la importancia de los resultados deportivos en la disposición anímica de las jugadoras, hecho constatado por otros estudios (Arroyo del Bosque et al., 2016; Pinilla-Nora, 2015), así como otras influencias contextuales, lo que sugiere la necesidad de un mayor control de variables. En este sentido, Martínez-Ferreiro (2016) señala que, aunque un entrenamiento que incide sobre la variable mental resulta de utilidad para cualquier deportista, independientemente de su nivel o edad, es posible que influyan multitud de factores de tipo deportivo o psicosocial en los resultados encontrados. El momento de la temporada en el que se encuentran, el numero de entrenamientos semanales, la situación en la clasificación en la que se encuentren durante el programa de intervención psicológica, problemas organizativos del club o entidad deportiva, etc.

En cualquier caso, cabe destacar el entrenamiento psicológico como una de las prácticas más importantes en el deporte de rendimiento, ya que tal y como indican Bradberry y Greaves (2012) respecto a la capacidad de entrenamiento de las funciones psicológicas, los neurólogos defienden la plasticidad o capacidad del cerebro para cambiar, por lo que el cerebro puede ser modificado, al igual que un músculo puede desarrollarse mediante el entrenamiento continuado. Y en este sentido, autores como Mora et al. (2001) subrayan que las capacidades psicológicas se pueden aprender, desarrollar y mejorar del mismo modo que ocurre con las capacidades físicas, por lo que los deportistas que emplean de forma más adecuada sus habilidades psicológicas pre- sentan una mayor probabilidad de efectuar un rendimiento óptimo (Bahrololoum et al., 2012; Del Pino, Gómez, Moreno y Gálvez, 2009).

Quizás la clave esté, no tanto en ver si el deportista o el equipo rinden mejor en el campo o en la pista, sino evaluar cómo ha mejorado sus recursos psicológicos, su habilidad mental con respecto al control de estrés, la reducción de la ansiedad, etc. En este sentido, como indican Olmedilla et al. (2010) la valoración del trabajo psicológico debe centrarse, básicamente, en la mejora del funcionamiento psicológico de los deportistas, aunque no se pueda, ni se deba, obviar su relación con el rendimiento deportivo. Y tal y como indican Page y Thelwell (2013) la "validación social", es decir la evaluación que realizan los sujetos sobre la formación recibida, es muy útil para determinar la satisfacción con la intervención.

Como limitaciones, quizás una de las más importantes sea la de ser un trabajo de campo, con deportistas en plena competición, lo que genera la influencia de variables contaminantes, que difícilmente pueden ser controladas. Por otro lado, el estudio hubiese resultado más rico si la muestra de equipos hubiese sido mayor, aunque, en ocasiones, resulta complicado obtener la colaboración de entrenadores, absolutamente necesaria, por los prejuicios que todavía hoy algunos de éstos. A veces creen que este tipo de programas pueden interferir en el día a día del entrenamiento deportivo, cuando realmente aquél debería estar inmerso en éste.

Por otra parte, la edad de los participantes y su implicación con la práctica deportiva pueden afectar al tratamiento. Así, el GE1 está formado por jugadoras muy jóvenes, con una edad media de 18 años y una experiencia de 10 años, lo que podría haber supuesto una mejor disposición general hacia el aprendizaje psicológico, lo que podría explicar, en parte, el aumento en las puntuaciones de todas las escalas, máxime cuando su objetivo deportivo era la permanencia, lo que se consiguió. El GE2 con más experiencia (casi 13 años de práctica continuada) y mayor edad, 21.6 años de media, participaban con mayores objetivos deportivos, lo que es posible que afectara a las puntuaciones de $\mathrm{MO}, \mathrm{HM}$ y $\mathrm{CH}$, mientras que el aumento significativo de las puntuaciones en CE y IER quizá represente la mejora tanto en el control de estrés como en el impacto de la evaluación de su rendimiento. Por último, el GC era el equipo de mayor edad (una media de 22.7 años) y mayor experiencia (casi 14 años), lo que pueda explicar las mayores puntuaciones de partida del equipo, aunque al no recibir tratamiento éstas no solo no aumentaron (IER) sino que descendieron (CE, MO y HM); solo en $\mathrm{CH}$ se produjo un aumento que podría ser explicado por la coincidencia de varios años de las componentes del grupo y gran estabilidad en el club como institución. En cualquier 
caso, parece que no es lo mismo un trabajo con futbolistas profesionales, que con futbolistas no profesionales. Quizás en equipos con mayor compromiso deportivo de los jugadores, este aspecto se podría controlar mejor.

Por último, para futuros programas de entrenamiento, sería interesante tener en cuenta las siguientes consideraciones:

1. Trabajar con grupos más reducidos de futbolistas por sesión. De manera, que el entrenamiento psicológico fuese más exhaustivo, además esto permitiría adecuarse a situaciones concretas de cada equipo, con mayor detalle.

2. Implementar los programas de entrenamiento psicológico desde inicio de temporada como una parte más del entrenamiento deportivo.

3. Obtener la colaboración e implicación de los entrenadores para llevar a cabo estos programas y que adquieran verdadero protagonismo, es decir, que estén insertos en el plan de entrenamiento normal del equipo

\section{Referencias}

Abdullah, M. R., Musa, R. M., Maliki, A. B. H. M. B., Kosni, N. A. y Suppiah, P. K. (2016). Role of psychological factors on the performance of elite soccer players. Journal of Physical Education and Sport, 16(1), 170-176. https://doi.org/10.7752/jpes.2016.01027

Abenza, L., González, J., Reyes, L., Reyes, F., Blas, A. y Olmedilla, A. (2014). Descripción y evaluación del entrenamiento psicológico de una deportista de regata clase laser radial. Revista Iberoamericana de Psicología del Ejercicio y el Deporte, 9(1), 67-92. https://doi.org/10.1400/218736

Andersen, M. B. y Williams, J. M. (1988). A model of stress and athletic injury: Prediction and prevention. Journal of sport and exercise psychology, 10, 294-306. https://doi.org/10.1123/ jsep.10.3.294

Arroyo del Bosque, R., Irazusta, S. y González-Rodríguez, O. (2016). Impacto del resultado post-partido en el estado de ánimo en jóvenes jugadoras de fútbol. CAFD. Universidad del País Vasco.

Bahrololoum, H, Hassani, A. Reza, M. y Akbari, A. (2012). The Relationship between the Emotional Intelligence and Mental Skills in Iranian Elite Male Volleyball Players. International Journal of Academic Research in Business and Social Sciences, 2(7), 123130.

Bennett, J. y Maynard, I. (2016) Performance blocks in sport: Recommendations for treatment and implications for sport psychology practitioners. Journal of Sport Psychology in Action, 8(1), 60-68. https://doi.org/10.1080/21520704.2016.1227414

Bradberry, T. y Greaves, J. (2012). Inteligencia emocional 2.0. Estrategias para conocer y aumentar su coeficiente. Barcelona, España: Conecta.

Brawley, L. R., Rejeski, W. J. y Lutes, L. (2000). A group mediated cognitive behavioral intervention for increasing adherence to physical activity in older adults. Journal of Applied Biobehavioral Research, 5(1), 47-65. https://doi.org/10.1111/j.1751-9861.2000. tb00063.x
Cabrita, T., Rosado, A., de la Vega, R. y Serpa, S. (2014). Relaciones entre identidad atlética y personalidad en el deporte de competición. Revista de Psicología del Deporte/Journal of Sport Psychology, 23(2), 247-253.

Cantón, E., Checa, I. y Ortin, F. J. (2009). Intervención psicológica con un nadador de medio acuático abierto. Cuadernos de Psicología del Deporte, 9(2), 57-65.

Castilla, J. F. y Ramos, L. C. (2012). Rendimiento deportivo, estilo de liderazgo y evitación experiencial en jóvenes futbolistas almerienses. Revista de Psicología del Deporte/Journal of Sport Psychology, 21(1), 137-142.

Del Pino, J., Gómez, E., Moreno, S. y Gálvez, G. (2009). Inteligencias múltiples y deporte. Apunts: Educación física y deporte, 95, 5-13.

Fernández-Fernández, R., Sánchez-Sánchez, L. y Zurita-Ortega, F. (2013). Eficacia de la hipnosis en la modificación de variables psicológicas y fisiológicas en deportistas. Universitas Psychologica, 12(2), 483-491. https://doi.org/10.11144/Javeriana. UPSY12-2.ehmv

García-Naveira, A. y Jerez, P. (2012). Departamento de psicología del club Atlético de Madrid: filosofía, programación y desempeño profesional en el futbol base. Cuadernos de Psicología del Deporte, 12(1), 111-120. https://doi.org/10.4321/S157884232012000100010

Gee, C., Dougan, R., Marshall, J. y Dunn, L. (2007). Using a normative personality profile to predict success in the National Hockey League (NHL): A 15-year longitudinal study. Journal of Sport \& Exercise Psychology, 29, S164.

Gimeno, F., Buceta, J. M. y Pérez-Llantada, M. C. (2007). Influencia de las variables psicológicas en el deporte de competición: evaluación mediante el cuestionario Características psicológicas relacionadas con el rendimiento deportivo. Psicothema, 19, 666-671.

González, J. (2017). Diseño del entrenamiento mental del tenista. De lo científico a la aplicado. Revista de Psicología Aplicada al Deporte y al Ejercicio Físico, 2, E5. https://doi.org/10.5093/rpadef2017a5

González-Campos, G., Valdivia-Moral, P., Zagalaz, M. L. y Romero, S. (2015). La autoconfianza y el control del estrés en futbolistas: revisión de estudios. Revista Iberoamericana de Psicología del Ejercicio y el Deporte, 10(1), 95-101.

Granero-Gallegos, A., Gómez-López, M., Abraldes, J. A. y Baena-Extremera, A. (2015). Predicción de las orientaciones de meta en el fútbol femenino. Journal of Sport and Health Research, 7(1), 31-42.

Gross, M., Moore, Z. E., Gardner, F. L., Wolanin, A. T., Pess, R. y Marks, D. R. (2016). An empirical examination comparing the mindfulness-acceptance-commitment approach and psychological skills training for the mental health and sport performance of female student athletes. International Journal of Sport and Exercise Psychology, 1-21. https://doi.org/10.1080/1612197X.2016.1250802

Guerrero, H. M., Saldaña, R. M. E. G., Bulnes, L.E., del Castillo, A. y Palencia, A. R. (2017). Intervención Cognitivo-Conductual en Jugadores Mexicanos de Fútbol Profesional. Cognitive-Behavioral Intervention in Mexican Professional Soccer Players. European Scientific Journal, ESJ, 13(8), 30-46. https://doi. org/10.19044/esj.2017.v13n8p30

Hofmann, S. G., Asnaani, A., Vonk, I. J., Sawyer, A. T. y Fang, A. (2012). The efficacy of cognitive behavioral therapy: A review 
of meta-analyses. Cognitive Therapy and Research, 36, 427-440. https://doi.org/10.1007/s10608-012-9476-1.

Jaenes, J. C., Caracuel, J. C. y Penaloza, R. (2012). Intervención en psicología del deporte: un caso de remo de alta competición. Revista de Psicología del Deporte/Journal of Sport Psychology, 21(1), 59-64.

Jones, G. (1993). The role of performance profiling in cognitive behavioral interventions in sport. The Sport Psychologist, 7(2), 160-172. https://doi.org/10.1123/tsp.7.2.160

Junge, A. y Feddermann-Demont, N. (2016). Prevalence of depression and anxiety in top-level male and female football players. BMJ Open Sport \& Exercise Medicine, 2(1). https://doi. org/10.1136/bmisem-2015-000087

Junichi, M. y Hajime, K. (2007). Psychological skill training for the Japanese soccer team in 2005 Universiade game in Izmir. Journal of Sport Science and Medicine, 6(10), 88.

Lindern, D. (2016). Desenvolvimento de uma intervenção com foco preventivo baseada na terapia cognitivo-comportamental e na psicologia positiva para atletas de futebol adolescentes. Tesis Dissertação de Mestrado. Portugal: Pontícia Universidade Católica do Rio Grande do Sul.

Llames, R. y García-Dantas, A. (2017). Entrenamiento psicológico deportivo aplicado a una estudiante de oposición. Revista de Psicología del Deporte/Journal of Sport Psychology, 26(Supl.4), 98-103.

Lorenzo, J., Pujals, C., Navarro, R. y Lorenzo, A. (2012). Análisis de los efectos de un programa de intervención psicológica en jóvenes jugadores de baloncesto. Revista de Psicología del Deporte/Journal of Sport Psychology, 27(1), 43-48.

Mahamud, J., Tuero, C. y Márquez, S. (2005). Características psicológicas relacionadas con el rendimiento: comparación entre los requerimientos de los entrenadores y la percepción de los deportistas. Revista de Psicología del Deporte/Journal of Sport Psychology, 14(2), 237-251.

Mahoney, M.J. (2013). Cognitive skills and athletic performance. En P. C. Kendall y S. D. Hollon (eds.), Cognitive-behavioral interventions: Theory, research, and procedures, (pp. 423-442). New York: Academic Press.

Martínez-Ferreiro, J. (2016). Inteligencia emocional y rendimiento deportivo en el futbol femenino de alta competición. Tesis Doctoral. Universidad de Valladolid, Valladolid.

Martín-García, G. (2003). La mujer futbolista desde la perspectiva psicológica. Cuadernos de Psicología del Deporte, 3(2), 7-15.

McArdle, S. y Moore, P. (2012). Applying evidence-based principles from CBT to sport psychology. The Sport Psychologist, 26, 299310. https://doi.org/10.1123/tsp.26.2.299

Mora, J. A., Zarco, J. A. y Blanca, M. J. (2001). Atención-concentración como entrenamiento para la mejora del rendimiento deportivo en jugadores profesionales de fútbol. Revista de Psicología del Deporte/Journal of Sport Psychology, 10(1), 49-65.

Morris, T. (2000). Psychological characteristics and talent identification in soccer. Journal of Sports Sciences, 18(9), 715-726. https://doi.org/10.1080/02640410050120096

Mostaan, M., Sanatkaran, A. y Bahari, S. M. (2015). The Effects of Behavioral Intervention on Mood in Disabled Professional Basketball Players. Journal of Novel Applied Sciences, 4, 797-803.

Nagle, F. J., Morgan, X. P., Hellickson, R. O., Serfass, R. C. y Alexander, J. F. (1975). Sporting success traits in Olympic contenders.
Physician Sportsmedical, 3(3), 31-34. https://doi.org/10.1080/0 0913847.1975 .11948293

Olatunji, B. O., Cisler, J.M. y Deacon, B.J. (2010). Efficacy of cognitive behavioral therapy for anxiety disorders: a review of meta-analytic findings. Psychiatric Clinics, 33(3), 557-577. https:/l doi.org/10.1016/i.psc.2010.04.002

Olmedilla, A., Bazaco, M. J., Ortega, E. y Boladeras, A. (2011). Formación psicológica, satisfacción y bienestar percibido en futbolistas jóvenes. Revista Científica Electrónica de Psicología, 12, 221-237.

Olmedilla, A. y Domínguez-Igual, J. (2016). Entrenamiento psicológico para la mejora de la atención y la autoconfianza en un futbolista. Revista de Psicología Aplicada al Deporte y al Ejercicio Físico, 1(e4), 1-11. https://doi.org/10.5093/rpadef2016a4

Olmedilla, A., García-Alarcón, A. y Ortega, E. (in press). Relaciones entre lesiones deportivas y estrés en fútbol 11 y fútbol sala femenino. Journal of Sport and Health Research.

Olmedilla, A., García-Mas, A. y Ortega, E. (2017). Características psicológicas para el rendimiento deportivo en jóvenes jugadores de fútbol, rugby y baloncesto. Acción Psicológica, 14(1), 7-16. https://doi.org/10.5944/ap. 14.1.19249

Olmedilla, A., Ortega, E., Andreu, M. D. y Ortín, F. J. (2010). Programa de intervención psicológica en futbolistas: evaluación de habilidades psicológicas mediante el CPRD. Revista de Psicología del Deporte/Journal of Sport Psychology, 19(2), 249-262.

Olmedilla, A., Ortega, E., González, J. y Serpa, S. (2015). Psychological training in sailing: Performance improvement for the Olympic classification phase. Universal Journal of Psychology, 3, 122-131. https://doi.org/10.13189/ujp.2015.030404

Olmedilla, A., Rubio, V. J., Ortega, E. y García-Mas, A. (2017). Effectiveness of a stress management pilot program aimed at reducing the incidence of sports injuries in young football (soccer) players. Physical Therapy in Sport, 24, 53-59. https://doi. org/10.1016/i.ptsp.2016.09.003

Orlick, T. (2003). Entrenamiento mental. Cómo vencer en el deporte y en la vida gracias al entrenamiento mental. Barcelona, España: Paidotribo.

Page, J. y Thelwell, R. (2013). The Value of Social Validation in Single-Case Methods in Sport and Exercise Psychology. Journal of Applied Sport Psychology, 25(1), 61-71. https://doi.org/10.1080/ 10413200.2012 .663859

Pazo, C. I. y Sáenz-López, P. (2008). Elaboración de las dimensiones, categorías y códigos para el análisis de la formación de los jóvenes talentos de fútbol. Comunicación presentada en IV Congreso Internacional y XXV Nacional de Educación Física. Córdoba, España. Recuperado de http://www.uco.es/IVCongresolnternacionalEducacionFisica/congreso/Documentos/001-082-020-004-001.html

Pazo, C. I., Sáenz-López, P. y Fradua, L. (2012). Influencia del contexto deportivo en la formación de los futbolistas de la selección española de fútbol. Revista de Psicología del Deporte/Journal of Sport Psychology, 21, 291-299.

Pinilla-Nora, P. (2015). Análisis de la relación entre estados de ánimo y la carga psicológica en entrenamientos y competición en el fútbol femenino. Trabajo Fin de Grado, Universidad de Extremadura.

Reyes, M., Raimundi, M.J. y Gómez, L. (2012). Programa de entrenamiento en habilidades psicológicas en jugadoras de voleibol de alto rendimiento. Cuadernos de Psicología del Deporte, 12(1), 9-16. https://doi.org/10.4321/S1578-84232012000100001 
Romero, A. E., Zapata, R., García-Mas, A., Brustad, R. J., Garrido, R. y Letelier, A. (2010). Estrategias de afrontamiento y bienestar psicológico en jóvenes tenistas de competición. Revista de Psicología del Deporte/Journal of Sport Psychology, 19(1), 117-133.

Rutkowska, K. y Bergier, J. (2015). Psychological Gender and Emotional Intelligence in Youth Female Soccer Players. Journal of Human Kinetics, 47(1), 285-291. https://doi.org/10.1515/ hukin-2015-0084

Silva, J. M., Shultz, B. B., Haslam, R. W. y Murray, D. F. (1981). A psychophysiological assessment of elite wrestlers. Research Quarterly for Exercise \& Sport, 52, 348-358. https://doi.org/10.1515/ hukin-2015-0084

Torres-Luque, G., Hernández-García, R., Olmedilla, A., Ortega, E. y Garatachea, N. (2013). Fluctuación del Perfil de Estados de
Ánimo (POMS) en un periodo competitivo en judokas de élite. Revista de Psicología del Deporte/Journal of Sport Psychology, 22, 313-320.

Whelan, J. P., Mahoney, M. J. y Meyers, A. W. (1991). Performance enhancement in sport: A cognitive behavioral domain. Behavior Therapy, 22, 307-327. https://doi.org/10.1016/S00057894(05)80369-7

Williams, A. y Reilly, T. (2000). Talent identification and development in soccer. Journal of Sports Sciences, 18, 657 - 667. tps://doi.org/10.1080/02640410050120041

Williams, K.T. (2017). Physiological and psychological characteristics of first division female football players. Doctoral dissertation. University of Johannesburg. 